\title{
Frequency sweep of the field scattered by an inhomogeneous structure using method of moments and asymptotic waveform evaluation
}

Troelsen, Jens; Meincke, Peter; Breinbjerg, Olav

Published in:

Proceedings of IEEE Antennas and Propagation Society

International Symposium

Link to article, DOI:

10.1109/APS.2000.874601

Publication date:

2000

Document Version

Publisher's PDF, also known as Version of record

Link back to DTU Orbit

Citation (APA):

Troelsen, J., Meincke, P., \& Breinbjerg, O. (2000). Frequency sweep of the field scattered by an inhomogeneous structure using method of moments and asymptotic waveform evaluation. In Proceedings of IEEE Antennas and Propagation Society International Symposium (Vol. 3, pp. 1830-1833). IEEE.

https://doi.org/10.1109/APS.2000.874601

\section{General rights}

Copyright and moral rights for the publications made accessible in the public portal are retained by the authors and/or other copyright owners and it is a condition of accessing publications that users recognise and abide by the legal requirements associated with these rights.

- Users may download and print one copy of any publication from the public portal for the purpose of private study or research.

- You may not further distribute the material or use it for any profit-making activity or commercial gain

- You may freely distribute the URL identifying the publication in the public portal 


\title{
Frequency Sweep of the Field Scattered by an Inhomogeneous Structure Using Method of Moments and Asymptotic Waveform Evaluation
}

\author{
Jens Troelsen*, Peter Meincke, and Olav Breinbjerg \\ Department of Electromagnetic Systems \\ Technical University of Denmark, DK-2800 Kgs. Lyngby, Denmark
}

\begin{abstract}
1. Introduction
In many radar applications it is necessary to determine the scattering from an object over a wide frequency band. The asymptotic waveform evaluation (AWE), which is a moment matching (MM) technique, constitutes a method to this end. In general, MM techniques provide a reduced-order model of a function. Using AWE-MM a linear system is expanded in terms of a Taylor series to obtain a fast frequency sweep of the linear system [1]. Recently, the AWE was applied to method of moments (MoM) solutions of the electric-field integral equation [2] and the combined-field integral equation [3] to get a fast frequency sweep of the radar cross section of a homogeneous structure. Besides showing that the AWE technique constitutes a fast and accurate tool in computational electromagnetics, it was found that a transformation of the coefficients of the Taylor series into the coefficients of a rational Padé function increases the bandwidth considerably [2]. If the structure of interest is inhomogeneous the MoM solution must be dictated by an integral equation that takes the variation of the electric properties of the structure into account. To the knowledge of the authors the AWE technique has not previously been applied to a MoM solution based on this kind of integral equation. It is the purpose of this paper to investigate the use of the AWE technique as a tool to obtain a fast frequency sweep of the field scattered by an inhomogeneous structure. The investigations concern 2-dimensional (2-D) configurations and TM-polarization. Throughout the paper the time factor $e^{-i \omega t}$ is suppressed.
\end{abstract}

\section{Asymptotic Waveform Evaluation}

In this section the AWE is applied to a 2-D configuration to obtain a fast frequency sweep of the electric field scattered by an inhomogeneous structure. The structure, which is invariant in the $z$-direction, is embedded in a homogeneous background medium. The electric field in this medium due to the impressed source is referred to as the electric background field. The cross section of the structure in the $(x, y)$-plane is denoted by $S$. We consider configurations with permeability of both the background medium and the scattering structure equal to that of free space, $\mu_{0}$. We define the wave number at position $\boldsymbol{\rho}$ in the $(x, y)$-plane as $k(\boldsymbol{\rho})=\omega \sqrt{\mu_{0}(\epsilon(\boldsymbol{\rho})+i \sigma(\boldsymbol{\rho}) / \omega)}$ where $\epsilon(\boldsymbol{\rho})$ is the permittivity and $\sigma(\boldsymbol{\rho})$ is the conductivity. Outside the structure $k(\boldsymbol{\rho})=k_{b}$ where $k_{b}=\omega \sqrt{\mu_{0}\left(\epsilon_{b}+i \sigma_{b} / \omega\right)}$ is the wave number of the background medium. The electric properties of the structure are described by the object function, $O(\rho)=k^{2}(\rho)-k_{b}^{2}$, which is zero outside the structure. For $\mathrm{TM}_{z}$-polarization the scattered field, $E_{s}(\rho)$, defined as the difference between the total field, $E(\rho)$,

The Danish Technical Research Council is acknowledged for partial support of this work. 
and the background field, $E_{b}(\boldsymbol{\rho})$, is given by the 2-D integral equation

$$
E_{s}(\rho)=E(\rho)-E_{b}(\rho)=\int_{S} g\left(\rho, \rho^{\prime}\right) E\left(\rho^{\prime}\right) O\left(\rho^{\prime}\right) d S^{\prime},
$$

where the 2-D scalar Green function is $g\left(\rho, \rho^{\prime}\right)=\frac{i}{4} H_{0}^{(1)}\left(k_{b}\left|\rho-\rho^{\prime}\right|\right)$ with $H_{0}^{(1)}$ being the Hankel function of zeroth order and first kind. For the discretization of the integral equation (1) we now expand $E(\rho)$ using pulse functions. Testing with delta functions at the centers of the cells yields

$$
[\overline{\bar{I}}-\overline{\bar{G}}(\omega)] \bar{E}(\omega)=\bar{E}_{b}(\omega),
$$

where $\omega$ denotes the angular frequency and $\bar{E}(\omega)$ and $\bar{E}_{b}(\omega)$ are column vectors. The n'th element of the vectors are referred to as $E_{n}$ and $E_{b, n}$. The matrix $\overline{\bar{G}}(\omega)$ contains the coupling between the cells. The element in the n'th row and $\mathrm{p}$ 'th column of $\overline{\bar{G}}(\omega)$ equals

$$
g_{n, p}=\int_{S_{p}} g\left(\boldsymbol{\rho}_{n}, \boldsymbol{\rho}^{\prime}\right) O\left(\boldsymbol{\rho}^{\prime}\right) d S^{\prime},
$$

where $\rho_{n}$ is the n'th test point and $S_{p}$ denotes the area of the p'th cell. Next, we introduce the coupling matrix $\overline{\bar{Z}}(\omega)$ and write the linear system (2) as

$$
\overline{\bar{Z}}(\omega) \bar{E}(\omega)=\bar{E}_{b}(\omega), \quad \text { where } \overline{\bar{Z}}(\omega)=[\overline{\bar{I}}-\overline{\bar{G}}(\omega)] .
$$

The AWE technique approximates the electric field by the first $K+1$ terms of a Taylor series expansion about $\omega_{0}$. We have

$$
\bar{E}(\omega) \approx \sum_{q=0}^{K} \bar{M}_{q}\left(\omega-\omega_{0}\right)^{q} .
$$

The vectors $\bar{M}_{q}$ are determined by differentiating (4) with respect to $\omega$. We introduce the general notation $f^{(q)}(\omega)=\frac{d^{q}}{d \omega^{q}} f(\omega)$ and write $\bar{M}_{q}$ as

$$
\bar{M}_{q}=\overline{\bar{Z}}^{-1}\left(\omega_{0}\right)\left[\frac{\bar{E}_{b}^{(q)}\left(\omega_{0}\right)}{q !}-\sum_{p=0}^{q} \frac{\left(1-\delta_{p 0}\right) \overline{\bar{Z}}^{(p)}\left(\omega_{0}\right) \bar{M}_{q-p}}{p !}\right]
$$

where $\delta_{p 0}$ is the Kronecker delta and the elements of $\overline{\bar{Z}}^{(p)}(\omega)$ can be written as

$$
Z_{n, p}^{(q)}= \begin{cases}\delta_{n p}-g_{n, p} & q=0 \\ -\int_{S_{p}}\left[g\left(\boldsymbol{\rho}_{n}, \boldsymbol{\rho}^{\prime}\right) O\left(\boldsymbol{\rho}^{\prime}\right)\right\}^{(g)} d S^{\prime} & q>0\end{cases}
$$

where

$$
\left[g\left(\boldsymbol{\rho}_{n}, \boldsymbol{\rho}^{\prime}\right) O\left(\boldsymbol{\rho}^{\prime}\right)\right]^{(q)}=\sum_{i=0}^{q} \frac{q !}{i !(q-i) !} g\left(\boldsymbol{\rho}_{n}, \boldsymbol{\rho}^{\prime}\right)^{(i)} O\left(\boldsymbol{\rho}^{\prime}\right)^{(q-i)} .
$$


The $q^{\prime}$ th derivatives of $g\left(\rho_{n}, \rho^{\prime}\right)$ and $O\left(\rho^{\prime}\right)$ with respect to $\omega, g\left(\rho_{n}, \rho^{\prime}\right)^{(\boldsymbol{q})}$ and $O\left(\boldsymbol{\rho}^{\prime}\right)^{(q)}$, are expressed in terms of the derivatives of $g\left(\boldsymbol{\rho}_{n}, \boldsymbol{\rho}^{\prime}\right)$ with respect to $k_{b}$ and the derivatives of $k$ and $k_{b}$ with respect to $\omega$.

It was shown in $[2,3]$ that it is possible to increase the bandwidth obtained using the Taylor series by transforming the Taylor coefficients into the coefficients of a rational Padé function. By applying the Padé function the n'th component of $\bar{E}(\omega)$ is approximated by

$$
E_{\mathrm{n}}(\omega) \approx \frac{\sum_{i=0}^{L} a_{n, i}\left(\omega-\omega_{0}\right)^{i}}{\sum_{j=0}^{M} b_{n, j}\left(\omega-\omega_{0}\right)^{j}},
$$

where $a_{n, j}$ and $b_{n, j}$ are the coefficients of the Padé function. We define $b_{n, 0}=1$ for all values of $n$ and require $L+M=K$. The coefficients of the Padé function are determined by matching $(9)$ and the components of (5).

\section{Numerical Example}

We investigate the monostatic scattering by a quadratic inhomogeneous cylinder illuminated by an electric line current, see Figure 1 . The media are described by $\left(\epsilon_{1}, \sigma_{1}\right)=\left(\epsilon_{0}, 0.01 \mathrm{~S} / \mathrm{m}\right),\left(\epsilon_{2}, \sigma_{2}\right)=\left(5 \epsilon_{0}, 0 \mathrm{~S} / \mathrm{m}\right)$, and $\left(\epsilon_{3}, \sigma_{3}\right)=\left(\epsilon_{0}, 0.02 \mathrm{~S} / \mathrm{m}\right)$. Figure 2 shows the real and imaginary parts of the $z$-component of the scattered electric field, $E_{s, z}$, at the observation point as a function of the frequency. The MoM-reference solution is compared to a 7 th order Taylor series $(K=7)$ and a Padé function with $L=4$ and $M=3$. A total of 144 cells (unknowns) are used to generate the coefficients of the Padé function. The plotted components are normalized by the absolute value of the electric background field at the origin, $E_{0}$. The constant value of $E_{0}$ is evaluated at $200 \mathrm{MHz}$.

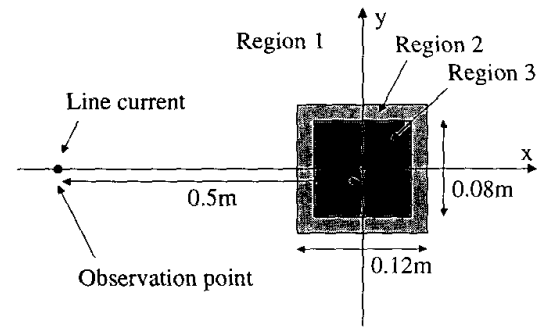

Figure 1: The axis of the quadratic cylinder coincides with the $z$-axis. The three regions are described by $\left(\epsilon_{1}, \sigma_{1}\right)=\left(\epsilon_{0}, 0.01 \mathrm{~S} / \mathrm{m}\right),\left(\epsilon_{2}, \sigma_{2}\right)=\left(5 \epsilon_{0}, 0 \mathrm{~S} / \mathrm{m}\right)$, and $\left(\epsilon_{3}, \sigma_{3}\right)=\left(\epsilon_{0}, 0.02 \mathrm{~S} / \mathrm{m}\right)$.

\section{Conclusions}

The AWE was used to obtain a fast frequency sweep of the field scattered by an inhomogeneous structure. By applying the AWE technique and using the Padé function with $\mathrm{L}=4$ and $\mathrm{M}=3$ a bandwidth of approximately $900 \mathrm{MHz}$ was obtained. The bandwidth can be further increased by increasing $L$ and $M$. It was found that the method is significantly faster than applying the ordinary MoM 
solution where the coupling matrix must be filled and inverted several times to cover the entire frequency band. The drawback of the method is the memory requirement since the coupling matrix and the derivatives of the matrix must be stored.
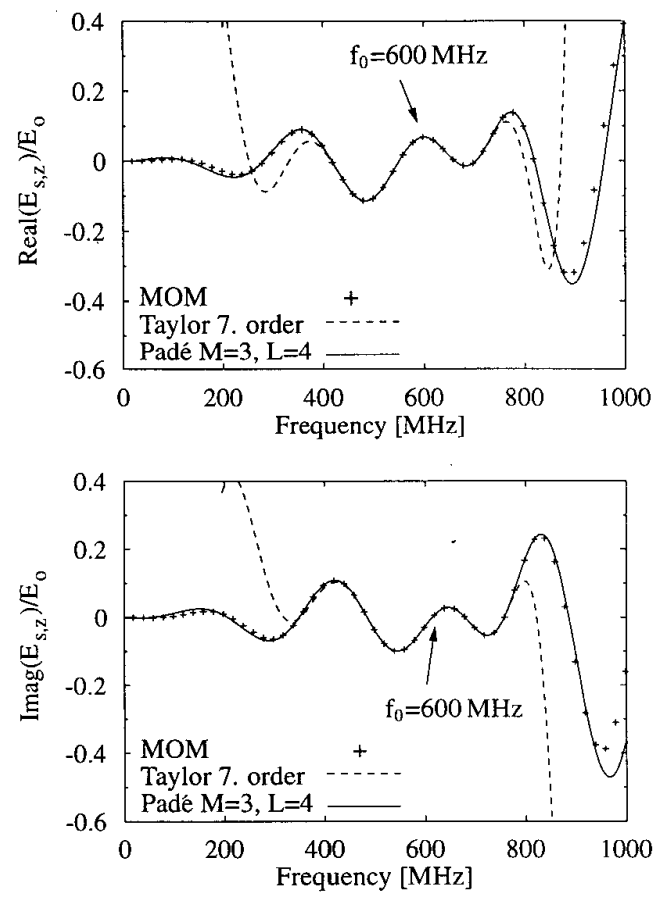

Figure 2: The real and imaginary parts of the scattered electric field for the monostatic case shown in Figure 1.

\section{References}

[1] E. Chiprout and N. S. Nakhla, Asymptotic Waveform Evaluation and Moment Matching for Interconnect Analysis, John Wiley \& Sons, 1994.

[2] C. J. Reddy, M. D. Deshpande, C. R. Cockrell, and F. B. Beck, "Fast RCS computation over a frequency band using method of moments in conjunction with asymptotic waveform evaluation technique," IEEE Trans. Antennas Propagat., vol. 46, pp. 1229-1233, Aug. 1998.

[3] D. Jiao, X. Y. Zhu and J. M. Jin, "Fast and accurate frequency-sweep using asymptotic waveform evaluation and the combined-field integral equation," Radio Science, vol. 34, pp. 1055-1063, Sept. - Oct. 1999. 